\title{
Fast Implementation of Transmit Beamforming for Colocated MIMO Radar
}

\author{
Gollakota Venkata Krishna SHARMA ${ }^{1}$, Konduri RAJA RAJESWARI ${ }^{2}$ \\ ${ }^{1}$ Dept. of ECE, GITAM Institute of Technology, GITAM University, India \\ ${ }^{2}$ Dept. of ECE, College of Engineering Autonomous, Andhra University, India
}

gvksarma@gitam.edu,krrau@yahoo.com

\begin{abstract}
Multiple-input Multiple-output (MIMO) radars benefit from spatial and waveform diversities to improve the performance potential. Phased array radars transmit scaled versions of a single waveform thereby limiting the transmit degrees of freedom to one. However MIMO radars transmit diverse waveforms from different transmit array elements thereby increasing the degrees of freedom to form flexible transmit beampatterns. The transmit beampattern of a colocated MIMO radar depends on the zerolag correlation matrix of different transmit waveforms. Many solutions have been developed for designing the signal correlation matrix to achieve a desired transmit beampattern based on optimization algorithms in the literature. In this paper, a fast algorithm for designing the correlation matrix of the transmit waveforms is developed that allows the next generation radars to form flexible beampatterns in real-time. An efficient method for sidelobe control with negligible increase in mainlobe width is also presented.
\end{abstract}

\section{Keywords}

Multiple-input multiple-output radar, transmit beamforming, fast implementation, waveform diversity, zero-lag correlation matrix

\section{Introduction}

MIMO radars employ multiple transmit antennas and multiple receive antennas thereby benefiting from increased degrees of freedom due to spatial diversity. These radars further have the flexibility to transmit diverse waveforms from their different transmit antennas thereby benefiting from waveform diversity. This spatial diversity and waveform diversity together can be used to improve many aspects of radar system performance [1]. MIMO configurations employing widely separated antennas [2] called "Statistical MIMO" radars improve target detection capabilities due to spatial diversity. MIMO radars can also employ closely spaced antennas [3] called "Colocated MIMO" radars to improve interference rejection capability, parameter identifiability and resolution performance due to increased virtual aperture. In this paper we deal with colocated MIMO radar only.

Conventionally MIMO radars employ orthogonal signals to obtain the phase delay for each transmitting/receiving antenna pair, thus increasing the degrees of freedom for beamforming. However MIMO radars can also employ partially correlated signals to form flexible transmit beampatterns with better performance than phased array beampatterns for a given number of transmit antenna elements. The advantages of MIMO radars (employing diverse waveforms) in forming flexible transmit beampaterns over phased array counterparts are well illustrated in [4]. While phased arrays employ only spatial diversity to form transmit beams, MIMO radars employ both spatial and waveform diversity to form transmit beams with enhanced flexibility.

The transmit beampattern of a colocated MIMO radar depends on the zero-lag correlation matrix of different transmit waveforms [5-6]. Orthogonal transmit waveforms result in uniform illumination of power in all directions which is very helpful in search applications. Fully correlated waveforms (perhaps also scaled by a complex constant) result in directive beams used in tracking applications. Partially correlated waveforms with a specific zerolag correlation matrix can be used to form a wide range of beampatterns.

To manage the complexity, the design of diverse waveforms to achieve a desired transmit beampattern is considered in the literature in two stages. In the first stage, the zero-lag correlation matrix $\mathbf{R}=\mathbf{X} \mathbf{X}^{\mathrm{H}}$ of the transmit waveform matrix $\mathbf{X}$ is designed. In the second stage, the design of signal waveform matrix $\mathbf{X}$ having a zero-lag correlation matrix $\mathbf{R}$ (obtained from the previous stage) and meeting some practically motivated constraints is considered. Low complexity algorithms for the latter problem exist in the literature [11-14]. Most of the existing algorithms in the literature addressing the former problem [4-10] are based on computationally complex optimization methods and hence difficult to solve in real-time. Design of signal correlation matrix for specific transmit beampattern was first addressed in [5], [6] as an optimization problem solved using gradient search method. In [4], the design of 
correlation matrix for designing different transmit beampatterns along with minimization of crossbeam pattern is formulated as an SDP optimization problem and solved using an efficient cyclic algorithm proposed therein. In [7], an eigenvalue decomposition method is used to achieve specific illumination pattern. Many target tracking applications require flexible transmit beampatterns generated in real-time. Previous works on signal correlation matrix design for approximation of desired transmit beampatterns employed optimization methods which are difficult to solve in real-time or highly complex to implement in hardware.

This paper considers the real-time computation of the zero-lag signal correlation matrix $\mathbf{R}$ that approximates a desired beampattern. The main contributions of this paper are (a) closed-form solution for computing the zero-lag correlation matrix and (b) FFT based algorithm for computing the zero-lag correlation matrix. The advantage of the proposed algorithm is that the proposed algorithm is easily amenable to hardware implementation since efficient architectures for FFT algorithms exist [18].

Section 2 describes MIMO radar signal model and defines a complete beampattern that includes the specification of transmit and cross beampattern. Section 3 derives the closed form solution and presents a fast implementation of the solution using 2D FFT algorithm. Section 4 presents the numerical results and Section 5 concludes the paper.

\section{MIMO Radar Signal Model}

Consider a monostatic MIMO radar that contains $M$ transmitters with the antenna elements configured as uniform linear arrays. We assume a point target and also that the target and transmitters lie in the same 2-D plane as shown in Fig. 1. Let $d_{\mathrm{T}}$ represent the spacing between consecutive transmitters. Let $\theta$ be the target angle with respect to the broadside direction and $\lambda$ is the carrier wavelength of the transmitted waveforms. Let $\left\{u_{m}(t)\right\}, m \in\{0,1, \ldots, M-1\}$ represent the $M$ transmitter waveforms. All the transmit antennas transmit waveforms simultaneously in time. We further assume that the transmitter waveforms are narrowband and the baseband signal waveforms are not modified because of Doppler effect [15]. The correlation between two transmit waveforms $u_{m}(t)$ and $u_{m},(t)$ at zero time-lag is defined as

$$
r_{m, m^{\prime}}=\int_{0}^{T_{0}} u_{m}(t) u_{m^{\prime}}^{*}(t) d t
$$

and $\mathbf{R}=\left[r_{m, m^{\prime}}\right]_{M \times M}$ represents the zero-lag correlation matrix of the $M$ transmit waveforms.

\subsection{Transmit Beampattern}

The baseband signal at the target location can be described by the expression

$$
\sum_{m=0}^{M-1} e^{-j 2 \pi f m} u_{m}(t) \triangleq \mathbf{a}^{H}(f) \mathbf{u}(t)
$$

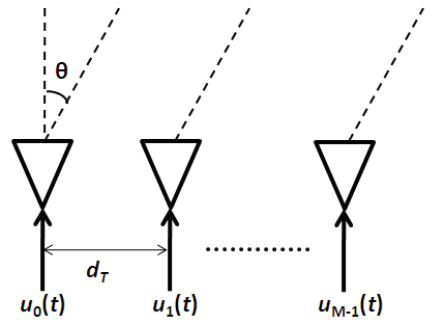

Fig. 1. Transmitter model.

where $f=d_{T} \sin (\theta) / \lambda$ is the spatial frequency of the target,

$$
\mathbf{u}(t)=\left[u_{0}(t) u_{1}(t) u_{2}(t) \ldots u_{M-1}(t)\right]^{T}
$$

is the vector of $M$ transmit waveforms and $\mathbf{a}(f)$ is the array steering vector given by

$$
\mathbf{a}(f)=\left[\begin{array}{llll}
e^{j 2 \pi f .0} & e^{2 \pi f .1} & \cdots & e^{j 2 \pi f(M-1)}
\end{array}\right]^{T} .
$$

With typical transmitter spacing of $d_{\mathrm{T}}=\lambda / 2$, the spatial frequency $f$ is in $[-1 / 2,1 / 2]$. The spatial distribution of power of the transmit signals is called the transmit beampattern and is given by [4],

$$
\begin{gathered}
P(f)=\mathbf{E}\left[\mathbf{a}^{H}(f) \mathbf{u}(t) \mathbf{u}^{H}(t) \mathbf{a}(f)\right]=\mathbf{a}^{H}(f) \mathbf{R a}(f) \\
=\sum_{m=0}^{M-1} \sum_{m^{\prime}=0}^{M-1} r_{m, m^{\prime}} e^{j 2 \pi f\left(m-m^{\prime}\right)} .
\end{gathered}
$$

Consider $P(f)$ for a phased array radar case. The $M \times 1$ transmit signal vector $\mathbf{u}(t)$ is given by $\mathbf{u}(t)=\mathbf{a}\left(f_{0}\right) u(t)$ where $f_{0}=d_{\mathrm{T}} \sin \left(\theta_{0}\right) / \lambda$ with $\theta_{0}$ denoting the steered direction. Then, $\mathbf{R}=\mathbf{a}\left(f_{0}\right) \mathbf{a}^{\mathrm{H}}\left(f_{0}\right)$ assuming unit power signal $u(t)$ and

$$
P(f)=\mathbf{a}^{H}(f) \mathbf{a}\left(f_{0}\right) \mathbf{a}^{H}\left(f_{0}\right) \mathbf{a}^{H}(f)=\left|\mathbf{a}^{H}(f) \mathbf{a}\left(f_{0}\right)\right|^{2} .
$$

Note that the transmit gain attains maximum value in the direction $\theta_{0}$ and is decreased at $\theta \neq \theta_{0}$. Now, consider $P(f)$ with orthogonal signals. Then, $\mathbf{R}=\mathbf{I}$, and

$$
P(f)=\mathbf{a}^{H}(f) \mathbf{a}(f)=M
$$

this implies that the beampattern is omnidirectional. Thus, the traditional beamforming results in a focused beampattern while the beampattern of MIMO with orthogonal signals is uniform in all directions. In some applications, it might be desirable to synthesize a beampattern that is between these two extremes so that wide focus areas can be formed without wasting power in the directions that are of no interest. This can be achieved by adjusting the correlation matrix $\mathbf{R}$ of the transmitted waveforms.

\subsection{Crossbeampattern}

The crosscorrelation between the signals backscattered to the radar by any two targets (at location parameters $f$ and $f^{\prime}$ ), called the crossbeampattern is given by 


$$
\begin{gathered}
c\left(f, f^{\prime}\right)=\mathbf{E}\left[\mathbf{a}^{H}(f) \mathbf{u}(t) \mathbf{u}^{H}(t) \mathbf{a}\left(f^{\prime}\right)\right]=\mathbf{a}^{H}(f) \mathbf{R a}\left(f^{\prime}\right) \\
=\sum_{m=0}^{M-1} \sum_{m^{\prime}=0}^{M-1} r_{m, m^{\prime}} e^{j 2 \pi\left(f m-f m^{\prime}\right)}, f \neq f^{\prime}
\end{gathered}
$$

In practical applications, it is desirable to minimize $c\left(f, f^{\prime}\right)$ for improving the quality of adaptive localization techniques [16]. In phased array beamforming, the signals backscattered to the radar from two targets (at location parameters $f$ and $f^{\prime}$ ) are fully coherent, which in particular makes the adaptive localization techniques inapplicable [16].

\subsection{Complete Beampattern}

The complete beampattern $s\left(f, f^{\prime}\right)$ as a function of spatial frequencies $f$ and $f^{\prime}$ 'can be written as

$$
s\left(f, f^{\prime}\right)=\mathbf{a}^{H}(f) \mathbf{R a}\left(f^{\prime}\right)=\sum_{m=0}^{M-1 M-1} \sum_{m^{\prime}=0} r_{m, m^{\prime}} e^{j 2 \pi\left(f m-f m^{\prime}\right)}
$$

We note that transmit beampattern $P(f)$ is the complete beampattern evaluated along the $f=f$ ' line i.e., $P(f)=s(f, f)$. The crossbeampattern $c\left(f, f^{\prime}\right)$ is the complete beampattern $s\left(f, f^{\prime}\right)$ at $f \neq f^{\prime}$. From (9), we see that the complete beampattern is the scaled two-dimensional Fourier transform of the zero-lag correlation matrix $\mathbf{R}$. We also notice that with typical transmitter spacing $d_{\mathrm{T}}=\lambda / 2, s\left(f, f^{\prime}\right)$ is periodic w. r. $\mathrm{t} f, f^{\prime}$ with period 1 .

\subsection{Problem Formulation}

The objective in transmit beampattern design, is to design $\mathbf{R}$ so that the transmit power is directed in desired directions $f$ and $c\left(f, f^{\prime}\right)$ is minimized. The specification of complete beampattern $s\left(f, f^{\prime}\right)$ (rather than just the transmit beampattern $P(f)$ captures both these goals. We state the design problem as follows: Given a desired beampattern function $s_{d}\left(f, f^{\prime}\right)$, find the matrix $\mathbf{R}=\left[r_{m, m},\right]_{M \times M}$ that closely approximates the relation

$$
s_{d}\left(f, f^{\prime}\right)=\sum_{m=0}^{M-1} \sum_{m^{\prime}=0}^{M-1} r_{m, m^{\prime}} e^{j 2 \pi\left(f m-f^{\prime \prime}\right)} .
$$

Any practically achievable signal correlation matrix $\mathbf{R}$ will be positive semi-definite and Hermitian (i.e. $\left.r_{m, m^{\prime}}=r_{m, m}^{*}\right)$. $\mathbf{R}$ will have the property if we choose the desired beampattern function $s_{d}\left(f, f^{\prime}\right)$ that is real valued and symmetric about the $f=f$ ' line. Further the total transmit energy can be constrained to $P$ by multiplying $\mathbf{R}$ by the factor $P / \operatorname{tr}\{\mathbf{R}\}$.

\section{Closed Form Solution}

We now proceed to derive a closed form solution to design the correlation matrix $\mathbf{R}$ to realize the desired complete beampattern $s_{d}\left(f, f^{\prime}\right)$ given by

$$
s_{d}\left(f, f^{\prime}\right)=\sum_{m=0}^{M-1} \sum_{m^{\prime}=0}^{M-1} r_{m, m^{\prime}} e^{j 2 \pi\left(f m-f^{\prime} m^{\prime}\right)} .
$$

Multiplying (11) on both sides by $e^{-j 2 \pi f k_{1}} e^{j 2 \pi f k_{2}}$ and integrating w.r.t $f$ and $f$ over one period $(0,1)$ we have

$$
\begin{aligned}
& \int_{0}^{1} \int_{0}^{1} S_{d}\left(f, f^{\prime}\right) e^{-j 2 \pi f k_{1}} e^{j 2 \pi f k_{2}} d f d f^{\prime} \\
& \quad=\int_{0}^{1} \int_{0}^{1} \sum_{m=0}^{M-1} \sum_{m^{\prime}=0}^{M-1} r_{m, m^{\prime}} e^{j 2 \pi f\left(m-k_{1}\right)} e^{-j 2 \pi f^{\prime}\left(m^{\prime}-k_{2}\right)} d f d f^{\prime} .
\end{aligned}
$$
we have

Interchanging the order of integration and summation

$$
\sum_{m=0}^{M-1} \sum_{m^{\prime}=0}^{M-1} r_{m, m^{\prime}}\left(\int_{0}^{1} e^{j 2 \pi f\left(m-k_{1}\right)} d f\right)\left(\int_{0}^{1} e^{-j 2 \pi f^{\prime}\left(m^{\prime}-k_{2}\right)} d f^{\prime}\right)=r\left(k_{1}, k_{2}\right)
$$

In the above equation, the relation $\int_{0}^{1} e^{-j 2 \pi f(n-k)} d f=\delta(n-k)$ has been used. The closed form solution for the zero-lag correlation matrix $\mathbf{R}=\left[r_{m, m}\right]_{M \times M}$ is given by

$$
r_{m, m^{\prime}}=\int_{0}^{1} \int_{0}^{1} s_{d}\left(f, f^{\prime}\right) e^{-j 2 \pi\left(f m-f^{\prime} m^{\prime}\right)} d f d f^{\prime} .
$$

A similar solution is presented in [17]. However the difficulty is in the evaluation of the integral in (14). In mission critical target tracking applications, computation of $\mathbf{R}$ from (14) is not possible in real-time. This problem is analogous to FIR filter design using Fourier series method wherein the desired unit sample response $h_{d}(n)$ of the FIR filter to the designed one is calculated from the desired frequency response $H_{d}\left(\mathrm{e}^{j \omega}\right)$ as [18].

$$
h_{d}(n)=\frac{1}{2 \pi} \int_{-\pi}^{\pi} H_{d}\left(e^{j \omega}\right) e^{j \omega n} d \omega .
$$

An approximation to $h_{d}(n)$ can be obtained by sampling $H_{d}\left(\mathrm{e}^{j \omega}\right)$ and using the inverse discrete Fourier transform to compute

$$
\begin{gathered}
\tilde{h}_{d}(n)=\frac{1}{K} \sum_{k=0}^{K-1} H_{d}\left(e^{j(2 \pi / K) k}\right) e^{j(2 \pi / N) k n}, \\
0 \leq n \leq N-1
\end{gathered}
$$

where $N$ is the length of the FIR filter. If $K>>N, \tilde{h}_{d}(n)$ can be expected to be a good approximation to $h_{d}(n)$. In a similar way, the integral in (14) can be approximated as

$$
\begin{aligned}
\tilde{r}_{m, m^{\prime}}= & \frac{1}{K} \frac{1}{K} \sum_{k=0}^{K-1} \sum_{k^{\prime}=0}^{K-1} S_{d}\left(\frac{k}{K}, \frac{k^{\prime}}{K}\right) e^{-j 2 \pi\left(\frac{k m}{K}-\frac{k^{\prime} m^{\prime}}{K}\right)} \\
& 0 \leq m \leq M-1,0 \leq m^{\prime} \leq M-1 .
\end{aligned}
$$

If $K>>M, \tilde{r}_{m, m}$, can be expected to be a good approximation to $r_{m, m}$, (17) can be efficiently computed 
using 2-D inverse fast Fourier transform (FFT). This allows the correlation matrix to be computed in real-time in contrast to previously proposed optimization based methods. The steps of the algorithm are presented below

1. Given the desired complete beampattern function $s_{d}\left(f, f^{\prime}\right)$ (specified over a large grid of points over $f$ and

$\left.f^{\prime}\right) \quad$ as $\hat{s}_{d}\left(k, k^{\prime}\right)=s_{d}\left(f, f^{\prime}\right)$ at $\left(f, f^{\prime}\right)=\left(\frac{k}{K}, \frac{k^{\prime}}{K}\right)$ with $k, k^{\prime} \in\{0,1, . ., K-1)$ where $K>>M$. Typically $K=3 M$ is adequate.

2. Find the 2-D inverse fast Fourier transform of $\hat{s}_{d}\left(k, k^{\prime}\right)$ to obtain $\hat{S}_{\mathrm{d}}\left(m, m^{\prime}\right)$.

3. The desired correlation matrix can be obtained as $r_{m, m^{\prime}}=\hat{S}_{d}\left((-m)_{M}, m^{\prime}\right)$ over $0 \leq m, m^{\prime} \leq(M-1)$. Here ()$_{M}$ indicates modulo $M$ operation.

In Step 3 above, the reflection property of 2-D Fourier transform viz. If $x\left(n_{1}, n_{2}\right) \rightarrow X\left(\omega_{1}, \omega_{2}\right)$ then $x\left(n_{1},-n_{2}\right)$ $\rightarrow X\left(\omega_{1},-\omega_{2}\right)$, is used.

\section{Design Results}

Two scenarios are considered for the simulation. The first scenario considers the maximum power design at known target locations and the second scenario considers beampattern matching design.

\subsection{Maximum Power Design for Known Target Locations}

In practice, to obtain the prior knowledge about the target locations, orthogonal waveforms [19], 20] are used for MIMO probing. This is referred to as "initial probing". After we get the target location estimates with this probing and spatial spectrum estimation techniques [21], we can optimize the transmitted beampattern to direct the transmit power in these known target locations. We define a simple parameterized expression for the desired beampattern function. Assume that there are $K$ targets in the range of the radar, located at angles $\theta_{k}$, corresponding to spatial frequencies $\left\{f_{k}\right\}, k=0,1, \ldots, K-1$ with $f_{k}=d_{\mathrm{T}} \sin \left(\theta_{k}\right) / \lambda$. We would like to concentrate the energy in the vicinity of each target. The desired beampattern $s_{d}\left(f, f^{\prime}\right)$ is expressed as

$$
s_{d}\left(f, f^{\prime}\right)=\sum_{k=1}^{K} s_{d, k}\left(f, f^{\prime}\right) * g\left(f, f^{\prime}\right)
$$

where

$$
\begin{gathered}
s_{d, k}\left(f, f^{\prime}\right)= \begin{cases}1 & f=f^{\prime}=f_{k} \\
0 & \text { otherwise }\end{cases} \\
g\left(f, f^{\prime}\right)=\frac{1}{f_{x} f_{y} \sqrt{1-\rho^{2}}} \cdot \exp \left\{\frac{-1}{2\left(1-\rho^{2}\right)}\left[\frac{f^{2}}{f_{x}^{2}}-\frac{2 \rho f f^{\prime}}{f_{x} f_{y}}-\frac{f^{\prime 2}}{f_{y}^{2}}\right]\right\}
\end{gathered}
$$

and $*$ represents convolution.
The function $g\left(f, f^{\prime}\right)$ is used to reduce the sidelobe level in the resulting beampattern by providing smooth tapering on all sides. When $f_{x}=f_{y}$ and $0<\rho<1$ the contour of $g\left(f, f^{\prime}\right)$ reduces to an ellipse rotated by angle $\pi / 4$ i.e. along the line $f=f^{\prime}$. This choice makes $s_{d}\left(f, f^{\prime}\right)$ symmetric along the $f=f$ ' line, resulting in a zero-lag correlation matrix $\mathbf{R}$ that is positive semi-definite and Hermitian. The choice of $\rho$ affects the resolution along the $f=f$ ' line and $f \neq f$ ' directions. The choice of $\rho=0$ and $\rho=1$ makes the contour of desired beampattern a circle and ellipse respectively at each desired direction along the $f=f$ ' line. The function in (19) provides more degrees of freedom to control sidelobe levels compared to a tapering window approach used in [11]. The values $f_{x}, f_{y}$ can be increased so that energy in $\hat{S}_{d}\left(m, m^{\prime}\right)$ can be spread uniformly along $m, m$, so that $r_{m, m} \approx$ constant thus satisfying the uniform elemental power constraint [4]. As an example, the desired beampattern function for three targets located at $f_{0}=0.25$, $f_{1}=0.4$ and $f_{2}=0.75$ is shown in Fig. 2 . The other parameters are chosen as $f_{x}=f_{y}=0.02$ and $\rho=0.2$

The 2-D inverse FFT coefficients $\hat{S}_{d}\left(m, m^{\prime}\right)$ of the desired beampattern function are shown in Fig. 3. We see that most of the energy is contained in the coefficients with $0 \leq m \leq 15$ and $0 \leq m^{\prime} \leq 15$.

The beampattern obtained for different values of $M$ is shown in Fig. 4 . Note that $M=5$ does not provide enough degrees of freedom for synthesizing three distinct beams. We also note that $M=20$ does not offer distinct advantages over $M=15$. The transmit beampattern for different values of $M$ is shown in Fig. 5.

One of the practically motivated constraint is to have the transmit waveforms with uniform elemental power constraint to limit the transmit signals having wildly varying magnitudes thereby limiting signal distortion. The diagonal elements of the computed correlation matrix represent the power of the transmit signals emitted by various transmitters. With $\rho=0.4$, the spread of the energy in the resulting correlation matrix $\mathbf{R}$, is evenly distributed resulting in a maximum to minimum element ratio of 1.2 thus nearly satisfying the uniform elemental power constraint.

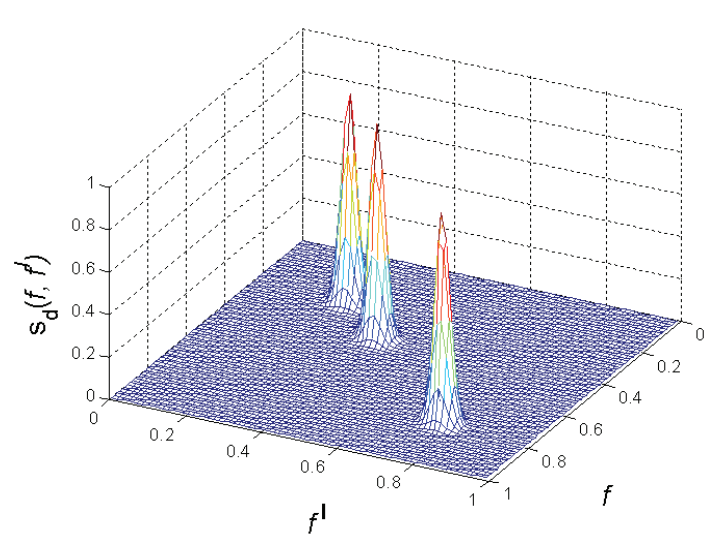

Fig. 2. Desired beampattern function $s_{d}\left(f, f^{\prime}\right)$. 


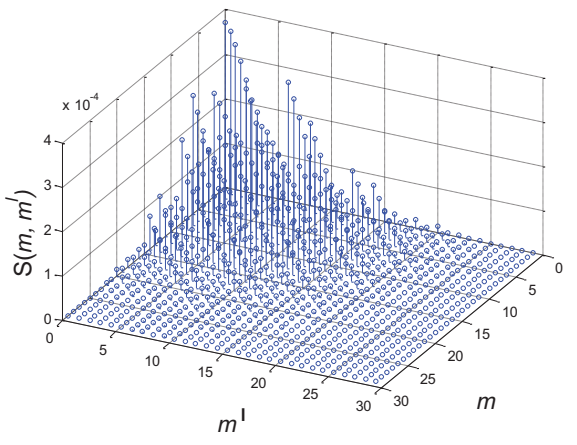

Fig. 3. 2-D inverse FFT coefficients $\hat{\mathrm{S}}_{\mathrm{d}}\left(m, m^{\prime}\right)$ of the desired beampattern function.
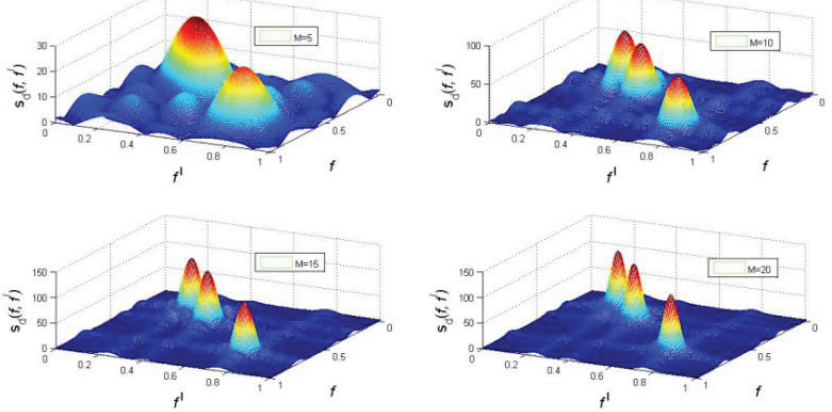

Fig. 4. Beampattern function with $M=5,10,15$ and 20 .
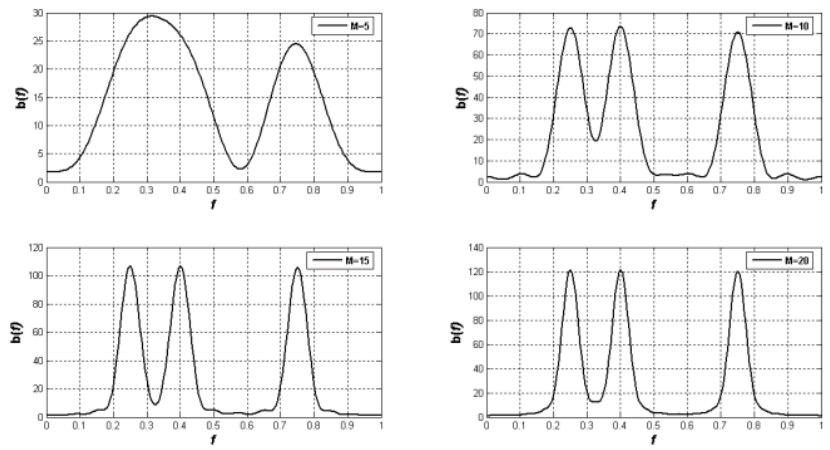

Fig. 5. Transmit beampattern with $M=5,10,15$ and 20 .

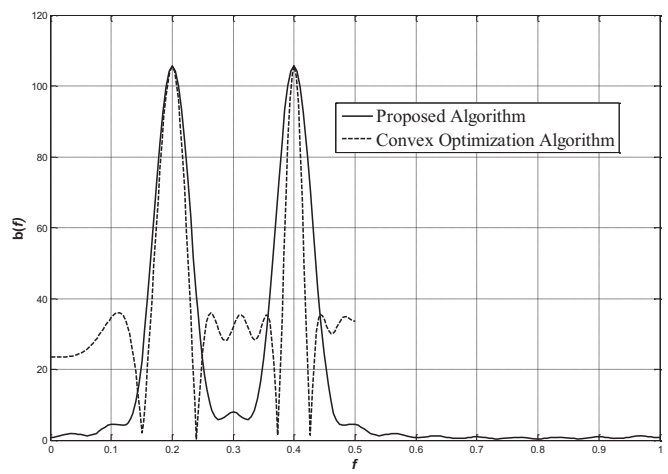

Fig. 6. Transmit beampattern evaluated using the algorithm in [4] and the proposed algorithm.

For comparison, the correlation matrices $\mathbf{R}_{i}$ and $\mathbf{R}_{i i}$ for achieving maximum power at spatial frequencies $f_{1}=0.2$ and $f_{2}=0.4$ are computed using (i) convex optimization method proposed in [4], and (ii) the proposed FFT based algorithm. The other parameters are chosen as $M=15, f_{x}=f_{y}=0.02$ and $\rho=0.2$. The transmit beampattern of corresponding correlation matrices is plotted in Fig. 6. It can be noticed that the proposed algorithm achieves the low sidelobe levels with small increase in mainlobe width.

\subsection{Beampattern Matching Design}

Consider a desired beampattern $\phi(\theta)$ given by

$\phi(\theta)=\left\{\begin{array}{cc}\mu_{k} & \theta \in\left[\theta_{k}-\Delta_{k}, \theta_{k}+\Delta_{k}\right], k=1,2, \ldots, K \\ 0 & \text { otherwise }\end{array}\right.$

with $K=3, \theta_{1}=-30^{\circ}, \theta_{2}=0^{\circ}, \theta_{3}=40^{\circ}, \Delta_{k}=10^{\circ}, \mu_{1}=0.5$, $\mu_{2}=1$, and $\mu_{3}=0.75$. Here $\theta_{k}$ represents the location of the $k^{\text {th }}$ target. $\Delta_{k}, \mu_{k}$ represent the beamwidth and transmit gain in the direction of the $k^{\text {th }}$ target respectively. The desired beampattern is obtained by convolution of ideal beampattern with Gaussian tapering given by (20) with $f_{x}=f_{y}=$ 0.015 and $\rho=0$. The desired beampattern for the three targets is shown in Fig. 7. The beampattern approximation for different values of $M$ is shown in Fig. 8. The transmit beampattern (in $\mathrm{dB}$ ) as a function of angle (in degrees) for different values of $M$ is shown in Fig. 9. From the above figures we notice that with $M=20$, the degrees of freedom are sufficient to closely approximate the desired beampattern. We also notice that $M=25$ does not offer improvement over $M=20$.

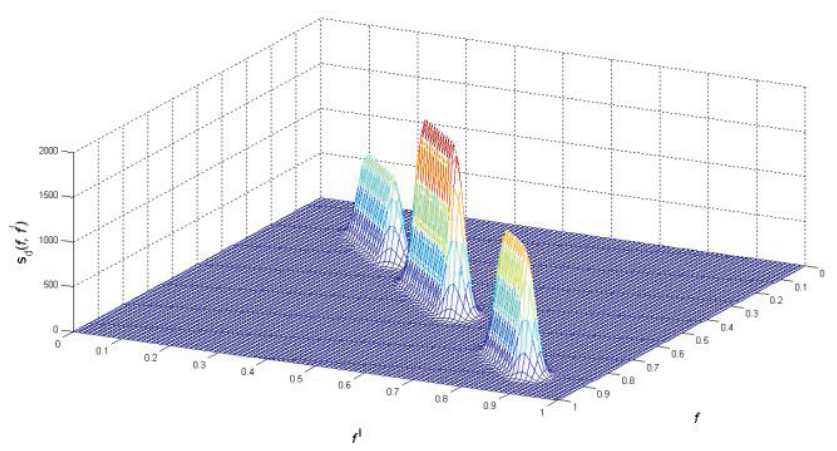

Fig. 7. Desired beampattern.
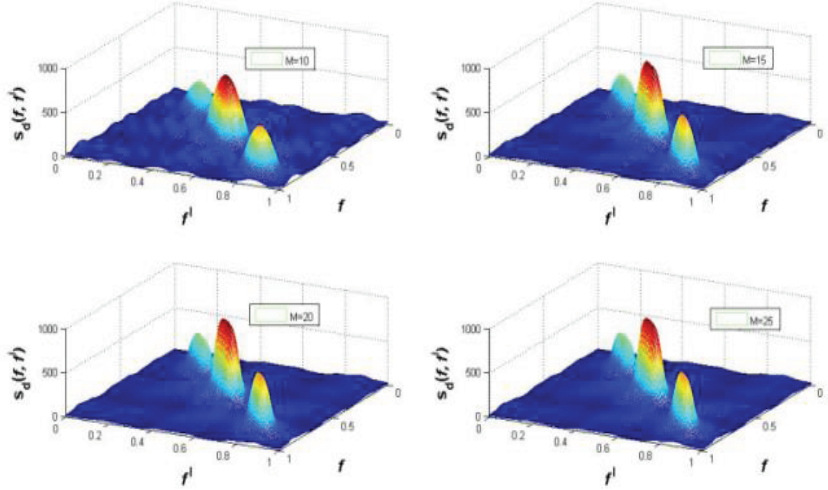

Fig. 8. Beampattern function with $M=10,15,20$ and 25 . 

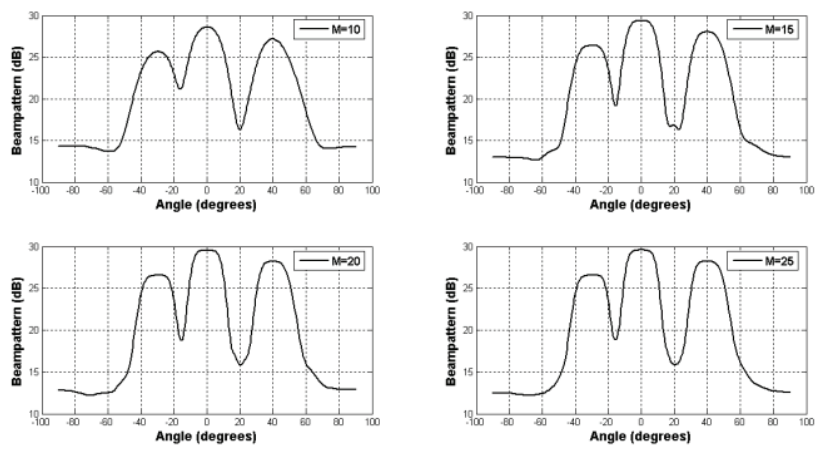

Fig. 9. Transmit beampattern with $M=10,15,20$ and 25 .

\subsection{Computational Complexity and Comparison with Previous Works}

FFT is the only operation utilized in the proposed algorithm. The complexity of the $N$-point FFT is known to be $O(N \log N)$ [18]. Since 2-D FFT algorithm is a separable transform, computing $\hat{S}_{d}\left(m, m^{\prime}\right)$ from $\hat{s}_{d}\left(k, k^{\prime}\right)$ in Step 2 of the algorithm above requires computing $M 1$-D FFTs along the rows of $\hat{s}_{d}\left(k, k^{\prime}\right)$ followed by $M$ 1-D FFTs along the columns. Hence, the overall complexity of the proposed algorithm is $O\left(M^{2} \log M\right)$. As a comparison, the SQP algorithms proposed in [4], [6], [8] have a complexity of $O\left(\log (1 / \eta) M^{3.5}\right)$ for an accuracy of $\eta$ [13]. For comparison, the number of computations required for calculating the above correlation matrix using (i) convex optimization method proposed in [4] and (ii) the proposed FFT based algorithm are evaluated using MATLABv7. The number of complex computations using both the algorithms (with $M=20, K=60$ grid points over $f, f \in$ ' $\{0,1\})$ are found to be 28317904 and 826712 respectively. Roughly, in this case the FFT based algorithm speeds up the computation by a factor of 34 .

In terms of the calculated transmit beampattern, it has been found that the solution given by the iterative methods [4-6], [9], [10] and the proposed algorithm in this paper achieve identical transmit beampatterns (under similar set of input conditions), with possibly a tradeoff between mainlobe width, sidelobe level and out-of-band roll off.

\section{Conclusions}

In this paper, a fast calculation of signal correlation matrix that approximates a desired transmit beampattern of colocated MIMO radar based on 2-D inverse FFT is proposed. Simulation results show correct operation of the proposed algorithm. While practically motivated constraints are easy to incorporate into the previously developed convex optimization based methods, they are difficult to solve in real time. However the proposed FFT based algorithm allows the correlation matrix to be computed in real-time and is easily amenable to hardware implementation [21].

Previous literature [1] considered windowing of zerolag correlation matrix to achieve low sidelobe level at the cost of increased mainlobe width. This paper achieved sidelobe control of beampattern function by considered the desired beampattern tapered by a two-dimensional Gaussian density function. Results show low sidelobe levels with negligible increase in mainlobe width. The sidelobe levels and mainlobe width of the crossbeam pattern can be controlled by adjusting the parameters of the function $g\left(f, f^{\prime}\right)$. If extremely low sidelobe levels are desired then the tapering windows approach [22] can be applied on the computed correlation matrix.

\section{References}

[1] LI, J., STOICA, P. (Eds.) MIMO Radar Signal Processing. New York: Wiley, 2008.

[2] HAIMOVICH, A. M., BLUM, R. S., CIMINI, L. J. MIMO radar with widely separated antennas. IEEE Signal Processing Magazine, 2008, vol. 25, no. 1, p. 116-129. DOI: 10.1109/MSP.2008.4408448

[3] LI, J., STOICA, P. MIMO radar with colocated antennas. IEEE Signal Processing Magazine, Sept. 2007, vol. 24, no. 5, p. 106 to 114. DOI: $10.1109 /$ MSP.2007.904812

[4] LI, J., STOICA, P., XIE, Y. On probing signal design for MIMO radar. IEEE Transactions on Signal Processing, Aug. 2007, vol. 55, no. 8, p. 4151-4161. DOI: 10.1109/TSP.2007.894398

[5] FUHRMANN, D., SAN ANTONIO, G. Transmit beamforming for MIMO radar systems using partial signal correlation. In Proc. 38th Asilomar Conference on Signals, Systems and Computers. Pacific Grove, (CA, USA), Nov. 2004, vol. 1, p. 295-299. DOI: 10.1109/ACSSC.2004.1399140

[6] FUHRMANN, D., SAN ANTONIO, G. Transmit beamforming for MIMO radar systems using signal cross-correlation. IEEE Transactions on Aerospace Electronic Systems, Jan. 2008, vol. 44, no. 1, p. 171-186. DOI: 10.1109/TAES.2008.4516997

[7] SHADI, K., BEHNIA, F. Transmit beampattern synthesis using eigenvalue decomposition in MIMO radar. In 8th International Conference on Information, Communication and Signal Processing (ICICS 2011). Singapore, December 2011. DOI: 10.1109/ICICS.2011.6174302

[8] AITTOMAKI, T., KOIVUNEN, V. Signal covariance matrix optimization for transmit beamforming in MIMO radars. In Conf. Record of the Forty-First Asilomar Conference on Signals, Systems and Computers (2007, ACSSC). Nov. 2007, p. 182-186. DOI: 10.1109/ACSSC.2007.4487191

[9] AHMED, S., THOMPSON, J. S., PETILLOT, Y. R., MULGREW, B. Unconstrained synthesis of covariance matrix for MIMO radar transmit beampattern. IEEE Transactions on Signal Processing, 2011, vol. 59, no. 8, p. 3837-3849. DOI: 10.1109/TSP.2011.2153200

[10] AITTOMAKI, T., KOIVUNEN, V. Low complexity method for transmit beamforming in MIMO radar. In Proc. IEEE International Conference on Acoustics, Speech, and Signal Processing (ICASSP). Honolulu (HI, USA), Apr. 2007, vol. 2, p. 305-308. DOI: 10.1109/ICASSP.2007.366233

[11] HE, H., STOICA, P., LI, J. Designing unimodular sequence sets with good correlations - including an application to MIMO radar. IEEE Transactions on Signal Processing, Nov. 2009, vol. 57, no. 11 , p. $4391-4405$. DOI: $10.1109 /$ TSP.2009.2025108

[12] LI, J., STOICA, P., ZHU, X. MIMO radar waveform synthesis. In IEEE Radar Conference 2008 (RADAR'08). Rome (Italy), May 2008, 6 p. DOI: 10.1109/RADAR.2008.4721118 
[13] AHMED, S., THOMPSON, J. S., PETILlOT, Y. R., MULGREW, B. Finite alphabet constant-envelope waveform design for MIMO radar. IEEE Transactions on Signal Processing, Nov. 2011, vol. 59, no. 11, p. 5326-5337. DOI: 10.1109/TSP.2011.2163067

[14] MATHELIER, B., KIRAN, D., S., REDDY, V. U. Synthesis of waveforms from zero-lag cross-correlation matrix with specified constraints and power levels. In International Conference on Signal Processing and Communications SPCOM 2012. Bangalore, 2012. DOI: 10.1109/SPCOM.2012.6289999

[15] PEEBLES, P. Z. Radar Principles. Wiley IEEE Press, 1998.

[16] XU, L., LI, J., STOICA, P. Radar imaging via adaptive MIMO techniques. In Proceedings of 14th European Signal Processing Conference (EUSIPCO'06). Florence (Italy), Sep. 2006.

[17] SRINIVAS, A., REDDY, V. U. Transmit beamforming for colocated MIMO radar. In International Conference on Signal Processing and Communications SPCOM. Bangalore, July 2010, 5 p. DOI: 10.1109/SPCOM.2010.5560551

[18] OPPENHEIM, A. V., SCHAFER, R. W. Digital Signal Processing. Prentice Hall, 1975.

[19] SHARMA, G.V.K., RAJA RAJESWARI, K. Four phase orthogonal code design for MIMO radar systems. In IEEE National Conference on Communications, NCC-2012. IIT Kharagpur (India), Feb. 2012, 4 p. DOI: 10.1109/NCC.2012.6176764

[20] RAO, H. M., SHARMA, G. V. K., RAJA RAJESWARI, K. Orthogonal phase coded waveforms for MIMO radars. International Journal of Computer Applications, Feb. 2013, vol. 63 , no. 6, p. 31-35. DOI: 10.5120/10471-5200
[21] WANHAMMAR, L. DSP Integrated Circuits. Academic Press, 1999.

[22] STOICA, P., MOSES, R. L. Spectral Analysis of Signals. Upper Saddle River, NJ: Prentice-Hall, 2005.

\section{About the Authors ...}

K. RAJA RAJESWARI is graduated in Electronics and Communication Engineering from Andhra University during 1976. She obtained her M.E. and Ph.D. also from the same university. Presently, she is a Professor in the Dept. of ECE, College of Engg., Andhra University, India. She is a senior member of IEEE. Her research interests include radar/sonar signal processing and wireless mobile communications. She has more than 100 papers published to her credit in reputed international/national journals and conferences.

G.V.K. SHARMA obtained his Ph.D. and Master's degree from Andhra University and Indian Institute of Science, Bangalore respectively. Presently, he is an Associate Professor in the Dept. of ECE, GITAM University, Visakhapatnam, India. His research interests include radar signal processing, VLSI signal processing and multicarrier communications. 